\title{
An Empirical Analysis of the Dog of the Dow Strategy for the Taiwan Stock Market
}

\author{
Hong Yan, Yu Song, Mingyue Qiu, and Fumio Akagi
}

\begin{abstract}
The Dog of the Dow investment strategy consists of buying the ten highest-dividend-yielding stocks in the Dow Jones Industrial Average in the American stock market and rebalancing the portfolio annually. The strategy is recognized as an effective method for portfolio selection in a lot of stock markets. In this study we apply the strategy to the Taiwan stock market (we name it Taiwan DoD strategy) and analyze its performance based on data from 2003 to 2012.

We propose here to buy the ten highest-dividend-yielding stocks in the Taiwan 50 Index and rebalance the portfolio annually. We conducted a simulation and found that the Taiwan DoD strategy always had a higher accumulated return than the Taiwan 50 Index. We also compared the risk-adjusted returns and investigate the performance of portfolios with less number of stocks. Simulation results show that the Taiwan DoD strategy is effective.
\end{abstract}

Index Terms - Dogs of the Dow strategy, investment strategy, portfolio selection, the Taiwan stock market, TAIEX, Taiwan 50 Index.

\section{INTRODUCTION}

Investors always seek ways to make profit or beat bench marks in stock markets. However, it is a difficult task even for professional fund managers.

The Dogs of the Dow investment strategy, also known as Dow 10 strategy, is a very simple dividend-based investment strategies. It was reported by Slatter in 1988 [1]. The strategy involves investing equal amounts in the 10 highest-dividend-yielding stocks of the Dow Jones Industrial Average (DJIA) index, and rebalancing the portfolio annually. The strategy is called the "Dogs of the Dow" because high-dividend-yielding stocks imply that the stocks are not approved by the market and hence are considered as dogs, i.e. losers in the market. Slatter reported that the Dogs of the Dow (DoD) strategy outperformed the bench marks such as DJIA and S\&P 500 from 1973 - 1988.

Similar results were reported in investment books like [2] and [3]. These books highlighted the Dogs of the Dow strategy and prompted its increasing popularity among institutional and individual investors.

In this paper, we examine the performance of the Dogs of

Manuscript received October 20, 2013; revised December 25, 2013.

H. Yan is with the Department of Management and Systems Engineering, Graduate School of Engineering, Fukuoka Institute of Technology, Fukuoka, Japan (e-mail: mdm12003@bene.fit.ac.jp).

Y. Song and F. Akagi are with the Department of System Management, Faculty of Information Engineering, Fukuoka Institute of Technology, Fukuoka, Japan (e-mail: \{song, akagi\} @ fit.ac.jp).

M. Qiu is with the Department of Intelligent Information System Engineering, Graduate School of Engineering, Fukuoka Institute of Technology, Fukuoka, Japan (e-mail: qmy1175116@126.com). the Dow strategy in a different market setting and a different time periods. In particular, our purpose is to analyze the performance of the Dogs of the Dow strategy in the Taiwan stock market. We implement simulations for data from 2002 to 2012 and compared the performance of Dogs of the Dow strategy with two popular market indices: the TAIEX (Taiwan Stock Exchange Capitalization Weighted Stock Index) and the Taiwan 50 Index (FTSE TWSE Taiwan 50 Index). We name the strategy Taiwan DoD strategy. We also simply refer it as DoD strategy in case that it clearly means Taiwan DoD strategy.

The results of simulations show that the DoD strategy outperforms the market indices both before and after risk-adjustment. And portfolios with fewer than ten stocks have even better performance than the original ten-stock DoD portfolio.

The remainder of this paper is organized as follows. In the next section, we review the related literature on the DoD investment strategy. Section III briefly introduces how to apply the strategy to the Taiwan stock market. Then based on the result of simulation, we compare the performance of the DoD with TAIEX and Taiwan 50 Index (Section IV). In Section V, we present the results for portfolios with fewer stocks and compare their performance with bench marks. To adjust higher-risk in DoD portfolios, we use the Sharp ratio to do adjustment and compare the performance with the benchmarks again (Section VI). Finally, Section VII concludes the paper with remarks on the future topics.

\section{RELATED LITERATURE}

\section{A. Studies on the American Market}

As mentioned above, the Dogs of the Dow investment strategy was first proposed for the American stock market. In [1], Slatter described that with the strategy; an investor selects the 10 highest-yielding stocks from the DJIA stocks at the end of each calendar year and invests equal amounts to each stock. After 1 year, the portfolio is rebalanced and updated with equally weighted investments in the new highest-yielding stocks. It was reported that from 1972 to 1987, the average annual return of such a portfolio outperformed DJIA by 7.6 percentage points.

For longer time horizons, O'Higgins and Downes [2] and Knowles and Petty [3] published books to introduce further information of the Dogs of the Dow strategy in the American market. In [2], the authors reported that the average annual return of the Dogs of the Dow is 6.2 points higher than DJIA during 1973 to 1991 period. Reference [3] showed that the Dogs of the Dow portfolio had an average annual return of $14.2 \%$, while the DJIA only had an average annual return 
$10.4 \%$ from 1957 to 1990 . They also examined an alternative version of the strategy in which the Dogs of the Dow portfolio consists of five highest-yielding stocks. The reported average annual return for this five dog stock strategy is $15.4 \%$, which is 1.2 points higher than the original ten-stock DoD portfolio.

The first academic study on the DoD strategy was performed by McQueen, Shields and Thorley [4]. They used statistical methods to examine the performance of the portfolio over 50 years (1946-1995), and concluded that this strategy's superiority is statistically significant.

\section{B. Studies on Other Markets}

Encouraged by its success in the American market, the Dogs of the Dow strategy has been examined in many other stock markets.

Reference [5] examined the Dogs of the Dow strategy in the British stock market. The authors simulated UK data from 1985 to 1994 and applied the Dogs of the Dow strategy to stocks included in the FTSE100 (Financial Times-Stock Exchange 100) index. The Dogs of the Dow portfolio was documented to outperform the market index on a risk adjusted basis in only 4 years out of 10 , thereby indicating that the Dogs of the Dow strategy was not particularly effective in the UK.

Andre and Silva studied its performance in Latin American stock markets from 1994 to 1999 . They found that the Dogs of the Dow slightly outperformed the market indices in Argentina, Chile, Colombia, Mexico, Peru and Venezuela, while the strategy seemed to underperform relative to market index in Brazil. Moreover, they conclude the result is lack of statistical significance, probably because of the short test period [6].

Effectiveness of the Dogs of the Dow strategy in the Canadian stock market was focused on the Toronto35 index during 1988-1997 and reported an average annual excess return $6.6 \%$ for the Dogs of the Dow portfolio. More importantly, the study showed that the Dogs of the Dow strategy produced significantly higher risk adjusted return than the Toronto35 and TSE300 (Toronto Stock Exchange 300) indices and the reported excess return were also high enough to compensate for the higher taxes and transaction costs [7].

In Japan, Song and Hagio proposed to apply the Dogs of the Dow strategy to Tokyo Stock Price Index 30 (TOPIX30) and NIKKEI 225 [8]. They showed that for data from 2002 to 2006, the Dogs of the Dow strategy is only slightly superior to the bench mark, while the performance is much better when applied to NIKKEI 225. Therefore they concluded that the strategy should be applied to NIKKEI 225 in the Japanese market. Qiu, Song and Hasama investigated the strategy for a longer period $(1981-2010)$. Simulation result showed the strategy outperformed the NIKKEI 225 and the result is statistical significant [9].

In [10], Rinne and Vahamaa summarized the performance of the Dogs of the Dow investment strategy in Aktiebolaget Optionsmäklarna / Helsinki Stock Exchange (OMX25) index from1998 to 2008. They can be successfully replicated in different types of markets and in different market conditions. Their result reported an annual abnormal return $4.5 \%$ and the outperformance of the strategy appeared particularly pronounced during stock market downtown.

Qiu, Yan and Song focused on Hong Kong stock market from 2001 to 2011 [11]. Based on the result of simulation, they reported that the Dogs of the Dow strategy outperformed the Hang Seng Index. However, the result was not statistically significant. They also found the portfolios with fewer than 10 dogs outperformed the bench mark. Thus, they concluded that the Dogs of the Dow strategy was effective in Hong Kong stock market.

For the Thai stock market, Tissayakorn, Song, Qiu and Akagi proposed applying the Dogs of the Dow strategy to the SET 50 Index. The simulation results showed that the strategy is also effective in the Thai stock market [12].

\section{APPLICATION TO THE TAIWAN StOCK MARKET}

\section{A. Taiwan Stock Market and Indices}

In this paper, we propose applying the Dogs of the Dow investment strategy to the Taiwan Stock Exchange (TWSE), which is the largest stock exchange of Taiwan district. There are more than 650 stocks listed at the market in 2013.

The most popular market index for the TWSE is the TAIEX (Taiwan Stock Exchange Capitalization Weighted Stock Index), which is calculated from the prices of all common stocks listed at TWSE. The index is a market capitalization-weighted price index which compares the current market value of all listed common shares with its value on the base of 1966 annual average, when the TAIEX was established and set at 100 points. In addition to the TAIEX, the TWSE also provides other indices to investors that include the Taiwan 50 Index (FTSE TWSE Taiwan 50 Index) and other sub-indices.

Here we apply the DoD strategy to Taiwan 50 Index which is "calculated from the stock prices of the top 50 listed companies on TWSE in terms of large market capitalization, high liquidity and compliance with requirements regarding the distribution of shares to minor shareholders" [13]. It is also a capitalization-weighted index, and was calculated from April 30, 2002 with a base value of 5000 points. The component stocks in the Taiwan 50 Index are reviewed quarterly in order to adjust for any changes that have occurred in the stock market, such as new listings or public offerings.

\section{B. Simulation}

We implement the simulation of Taiwan DoD strategy in the following steps.

1) Step 1: Collect data of all the 50 companies of Taiwan 50 Index on 31 November, and then selected the 10 highest dividend yielding stocks; invest in the 10 stocks with equally amount at the beginning of the next year.

2) Step 2: Hold these stocks for 1 year, and then sell them out at the end of the next year. After updating the list of Taiwan 50 Index, invest in the new top 10 stocks with equally weighting.

3) Step 3: Repeat the above process every year.

In this study, we conducted the simulation for the 2002-2012 data. In particular, we collected the first set of 
data at the end of 2002 and invested at the beginning of 2003. The simulation ended with liquidating the portfolio at the end of 2012. We searched for the price of the stocks from the Internet and obtained the dividend data from the TWSE [13, $14]$.

\section{Simulation Result}

Fig. 1 shows the annual returns of each year. Out of the 10 years, the annual returns of DoD were positive in 8 years. The greatest return was almost $100 \%$ (2009). The returns are negative in only 2 years. One of them was 2008, when the Lehman Shock hit all over the world.

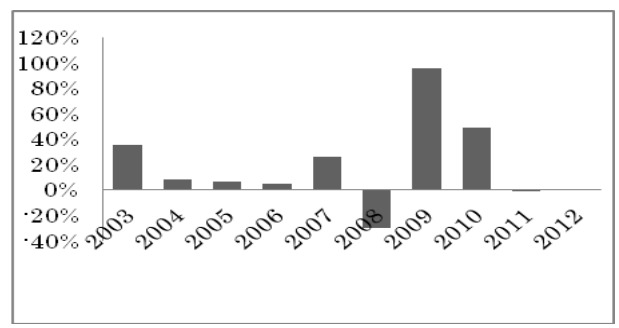

Fig. 1. Annual returns of the DoD strategy.

\section{COMPARISON OF PERFORMANCE}

In this section, we compare the performance of the Taiwan DoD strategy with TAIEX and the Taiwan 50 Index from various points of view.

\section{A. Annual Return}

Table I shows the average return and deviation of Taiwan DoD strategy, the TAIEX and the Taiwan 50 Index. We can see that the DoD strategy had an average return of $19.43 \%$ and a standard deviation of $32.62 \%$, while Taiwan 50 Index portfolio had a lower mean return and deviation of $9.24 \%$ and $28.90 \%$, respectively, and those of TAIEX were $10.10 \%$ and $30.69 \%$, respectively.

TABLE I: ANNUAL RETURN SUMMARY STATISTICS (2002 TO 2012)

\begin{tabular}{|c|c|c|}
\hline & $\begin{array}{c}\text { Average } \\
\text { Return }\end{array}$ & Standard Deviation \\
\hline Taiwan DoD & $19.43 \%$ & $32.62 \%$ \\
\hline Taiwan 50 & $9.24 \%$ & $28.90 \%$ \\
\hline TAIEX & $10.10 \%$ & $30.69 \%$ \\
\hline
\end{tabular}

TABLE II: DIFFERENCE BETWEEN DOD AND BENCH MARKS (UNIT: PERCENTAGE POINTS)

\begin{tabular}{|c|c||c|}
\hline \hline & $\begin{array}{c}\text { Average } \\
\text { Return }\end{array}$ & Standard Deviation \\
\hline \hline Taiwan 50 & 10.19 & 3.72 \\
\hline \hline TAIEX & 9.33 & 1.93 \\
\hline
\end{tabular}

Table II illustrates data on the difference between the Taiwan DoD portfolios and the bench marks. Comparing with the Taiwan 50 Index, the DoD strategy had a 10.19 percentage points higher return on average, and the difference of the standard division was 3.72 percentage points. For TAIEX, the difference was 9.33 and 1.93, respectively.

From the result, we can see the average return of Taiwan DoD strategy was near twice of the benchmarks while they had alike standard deviation (risk). Hence we conclude that the Taiwan DoD outperformed both the TAIEX and the Taiwan 50 Index in the 10 years on average.

\section{B. Differences between Annual Return}

Fig. 2 plots the annual returns of the DoD strategy, Taiwan 50 Index and TAIEX portfolios for each of the 10 years.

From Fig. 2, we can see that the Taiwan DoD strategy portfolio outperformed 7 times than the Taiwan 50 Index, and outperformed 8 times than the TAIEX from 2002 to 2012. Especially in 2009, the DoD strategy was about 40 percentage points greater than the benchmarks. In 2008 and 2011, though the annual returns of the DoD were negative, they were superior to the benchmarks largely. Hence the Taiwan DoD strategy seems to be quite robust in recession.

\section{Accumulated Performance}

Fig. 3 demonstrates the accumulated performance of the DoD strategy, the Taiwan 50 Index and the TAIEX. From 2002 through 2012, the DoD strategy always had a higher accumulated return than the Taiwan 50 Index and the TAIEX, especially after 2009.

In 2012, DoD strategy had an accumulated value of $415.77 \%$, which is more than 4 times the value in 2002 . This means that if you had invested 100 Taiwan dollars at the end of 2002, the return would be 315.77 Taiwan dollars at the end of 2012. On the contrary, the value of the Taiwan 50 Index in 2012 was only $164.87 \%$, which is about 1.5 times of the data of 2002. And the accumulated value of the TAIEX was quite similar with that of the SET Index.

Therefore, we conclude that in middle term, the Taiwan DoD strategy is very powerful to make profit and beat the bench marks.

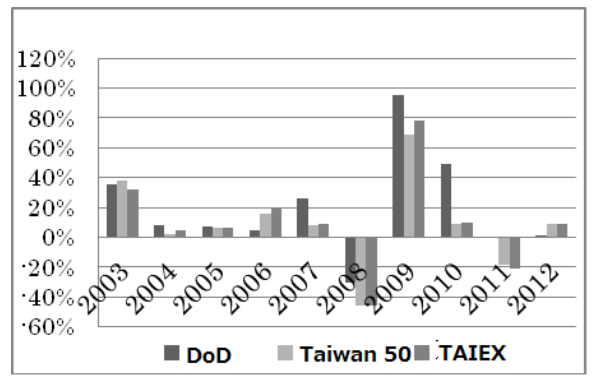

Fig. 2. Annual returns of the DoD strategy, TAIEX, and Taiwan 50 Index.

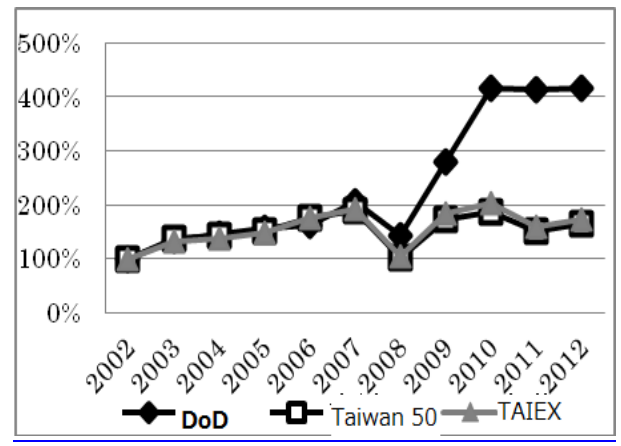

Fig. 3. Accumulated performances.

\section{PORTFOlios With FEWER NUMBER OF STOCKS}

In this section, we test other portfolios with less than 10 stocks. We named the portfolio with the top N stocks as Dow 
$\mathrm{N}$ strategy. The simulation of these strategies was conducted in a manner similar to that of the Taiwan DoD strategy but by using the top $\mathrm{N}$ stocks instead of the top-10 stocks.

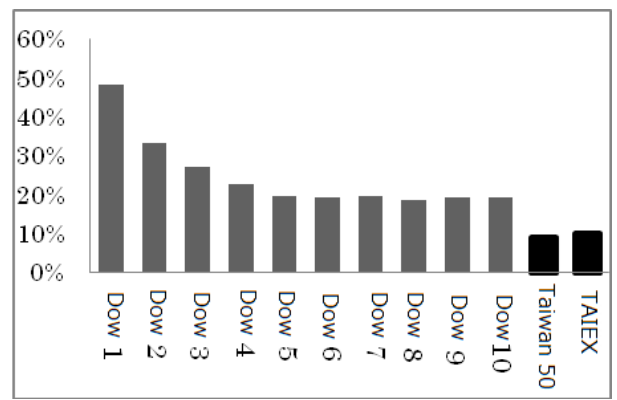

Fig. 4. Average returns of various portfolios.

From Fig. 4, we can conclude that all portfolios of the Dogs of the Dow investment strategy outperformed Taiwan 50 Index during 2002-2012 period. The Dow 1 portfolio had the highest average annual return $(48.35 \%)$. This is almost 5 times of the benchmarks. The second best was the Dow 2 portfolio with a value of $33.43 \%$. For portfolios with 5 or more stocks, there were no significant differences.

In conclusion, using Taiwan DoD strategy, even portfolios with less numbers of stocks could outperform the benchmarks, especially portfolios with less than 4 stocks.

\section{RISK ADJUSTMENT}

Although analysis of Table I showed that the Taiwan DoD had a higher mean return than the benchmarks, it also showed that DoD strategy had higher standard deviations (i.e. risk) than the benchmarks. With only 10 stocks in the portfolio, there are some unsystematic risks leads to the higher standard deviations. Hence we need to adjust the risk of DoD portfolios, and it will be clearer for us to see the results.

Sharpe Ratio is a well-known technique used in risk-adjustment. After calculating the two different degree risk portfolios by Sharpe Ratio, we can more exactly judge the performance of the two different strategies.

Here we use 1-year Taiwan government loan [15] as the risk free rate. The third column of Table III displays the risk-adjusted average returns of Dow $\mathrm{N}$.

TABLE III: AVERAGE RETURNS BEFORE AND AFTER RISK-ADJUSTMENT

\begin{tabular}{|c|c|c|}
\hline & $\begin{array}{c}\text { Average returns } \\
\text { before adjustment }\end{array}$ & $\begin{array}{c}\text { Risk-adjusted average } \\
\text { returns }\end{array}$ \\
\hline Dow 1 & $48.35 \%$ & $19.50 \%$ \\
\hline Dow 2 & $33.43 \%$ & $19.87 \%$ \\
\hline Dow 3 & $27.27 \%$ & $18.43 \%$ \\
\hline Dow 4 & $22.58 \%$ & $17.89 \%$ \\
\hline Dow 5 & $19.48 \%$ & $17.02 \%$ \\
\hline Dow 6 & $19.28 \%$ & $16.00 \%$ \\
\hline Dow 7 & $19.49 \%$ & $16.76 \%$ \\
\hline Dow 8 & $18.52 \%$ & $16.76 \%$ \\
\hline Dow 9 & $19.28 \%$ & $17.63 \%$ \\
\hline Dow 10 & $19.43 \%$ & $18.37 \%$ \\
\hline
\end{tabular}

After risk adjustment, the average returns of Dow $\mathrm{N}$ portfolios were between $16.00 \%$ and $19.87 \%$. The differences between Dow $\mathrm{N}$ portfolios were much less than those before adjustment. Nevertheless, they still outperformed Taiwan 50 Index and TAIEX by more than 5 points. Hence we may conclude that Taiwan DoD strategy is an effective strategy even after risk-adjustment.

\section{CONCLUSION}

In this study, we proposed applying the Dogs of the Dow investment strategy to the Taiwan stock market and compared the performance of the Taiwan DoD strategy with bench marks on the basis of the results of a simulation. We found that although the Dogs of the Dow strategy performed poor in a few years, it outperformed the Taiwan 50 Index and the TAIEX in the Taiwan stock market on average.

We also found that the portfolios with fewer than ten stocks outperformed the benchmarks both before and after risk-adjustment. Therefore, we can conclude that in middle term, the Taiwan DoD strategy is effective enough to make profit and outperform the benchmarks in the Taiwan stock market

In this paper, we have not adjusted transaction costs and tax in our simulations. As a future work, we need to take these costs into account and examine the simulation result under the new conditions.

\section{REFERENCES}

[1] J. Slatter, "Study of industrial average's finds stocks with high dividends are big winners," Wall Street Journal (Eastern edition), August 11, 1988.

[2] M. O. Higgins and J. Downes, Beating the Dow, Harper Perennial, New York, 1991.

[3] H. C. Knowles and D. H. Petty, The Dividend Investor, A Safe and Sure Way to Beat the Market with High-Yield Dividend Stocks, Probus Publishing, Chicago, 1992.

[4] G. Queen, K. Shields, and S. Thorley, "Does the 'Dow-10 investment strategy' beat the Dow statistically and economically?" Financial Analysts Journal, vol. 53, no.4, pp. 66-72, 1997.

[5] S. Visscher and G. Filbeck, "Dividend yield strategies in the British stock market," The European Journal of Finance, vol. 3, no. 4, pp. 227-289, 1997.

[6] L. C. Andre and D. Silva, "Empirical test of the Dogs of the Dow strategy in Latin American stock markets," International Review of Financial Analysis, vol. 10, no. 2, pp. 187-199, 2001.

[7] S. Visscher and G. Filbeck, "Dividend-yield strategies in the Canadian stock market,” Financial Analysts Journal, vol. 59, pp. 99-106, 2003.

[8] Y. Song and K. Hagio, "A study on portfolio selection strategies for stock investment," Research Bulletin of Fukuoka Institute of Technology, In Japanese, vol. 40, no. 1, pp. 29-36, 2007.

[9] M. Qiu, Y. Song, and M. Hasama, "Empirical analyses of the Dog of the Dow strategy: Japanese evidence," International Journal of Innovative Computing, Information and Control, vol. 9, pp. 3677-3687, 2013.

[10] E. Rinne and S. Vahamaa, "The 'dogs of the Dow' strategy revisited: Finnish evidence," The European Journal of Finance, vol. 17, no. 5-6, pp. 451-469, May-July 2011.

[11] M. Qiu, H. Yan, and Y. Song, "Empirical analyses of the dogs of the Dow strategy: Hong Kong evidence," European Journal of Management, vol. 12. no. 3, pp. 183-187, 2012.

[12] K. Tissayakorn, Y. Song, M. Qiu, and F. Akagi, "A study on effectiveness of the dogs of the Dow strategy for the Thai stock investment," International Journal of Innovation, Management and Technology, vol. 4, pp. 277-280, 2013.

[13] Homepage of Taiwan Stock Exchange. [Online]. Available: http://www.twse.com.tw/ch/index.php.

[14] The price and dividend of Taiwan 50 Index stocks. [Online]. Available: http://www.cnyes.com/twstock/

[15] Homepage of Bank of Taiwan. [Online]. Available: http://www.bot.com.tw/Pages/default.aspx. 
Hong Yan was born in China. She earned her bachelor degree at Fukuoka Institute of Technology, Japan in 2012. Currently she is a master degree student in Department of Management and System Engineering, Graduate School of Engineering, Fukuoka Institute of Technology, Fukuoka, Japan.

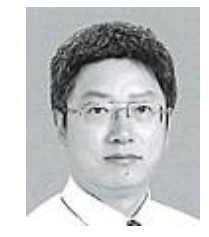

Yu Song was born in China. He earned his Ph.D. at Tohoku University, Japan. Currently he is a professor at Department of Systems Engineering, Fukuoka Institute of Technology, Fukuoka, Japan. His research interests include financial engineering, queueing theory and other operations research techniques. Prof. Song is a senior member of IEDRG.

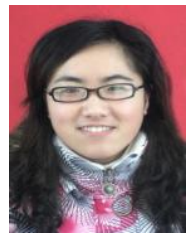

Mingyue Qiu was born in China. She earned her master degrees at both Fukuoka Institute of Technology, Japan and Nanjing University of Science and Technology, China in 2012. Currently she is a Ph. D. student at Graduate School of Engineering, Fukuoka Institute of Technology, Fukuoka, Japan.

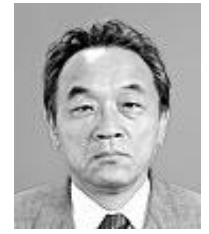

Fumio Akagi was born in Japan. He earned his Ph.D. at Osaka City University, Japan in 1985. Currently he is a professor at Department of Systems Engineering, Fukuoka Institute of Technology, Fukuoka, Japan. His research interests include financial engineering and production management. Prof. Akagi is a member of Japan Industrial Management Association. 